\title{
Caffeine Enhances the Balance System and Postural Balance in Short Time in Healthy Individuals
}

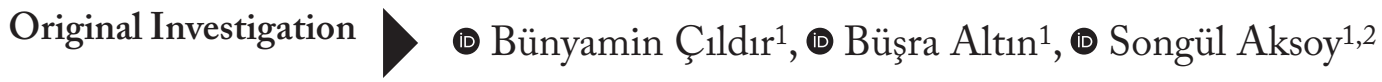 \\ ${ }^{1}$ Department of Audiology, Hacettepe University Faculty of Health Sciences, Ankara, Turkey, \\ ${ }^{2}$ Dizziness and Balance Disorders Research and Application Center, Hacettepe University, Ankara, Turkey
}

\begin{abstract}
Objective: This study aimed to explore the effects of caffeine on balance function by determining the extent to which caffeine consumption affects postural sway and balance control in healthy individuals.

Methods: Thirty healthy participants aged 20-35 years without any vestibular disorder were enrolled in this study. The participants were randomly divided into two groups, and those in Group 1 were given two cups of regular coffee (300-350 mg) while those in Group 2 were given two cups of decaffeinated coffee. The sensory organization test, the head shake sensory organization test, the limits of stability test, and the adaptation test were performed on all participants before and after coffee intake using computerized dynamic posturography.

Results: The sensory organization test composite scores $(\mathrm{p}=0.001)$ and the head shake condition 5 (C5) equilibrium scores ( $\mathrm{p}=0.001$ ) of the participants in Group 1 showed a statistically significant increase after coffee intake while the composite scores $(\mathrm{p}=0.001)$ and the head shake condition (C5) equilibrium scores ( $\mathrm{p}=0.001$ ) of those in Group 2 showed a statistically significant decrease. There was a statistically significant difference between the two groups in the equilibrium scores in the pitch plane $(\mathrm{p}=0.001)$, the yaw plane $(\mathrm{p}=0.001)$, and the roll plane $(\mathrm{p}=0.001)$ of $\mathrm{C} 5$ after coffee intake.

B.C.. 0000-0003-2962-6309;

B.A. 0000-0002-7032-5658;

S.A. 0000-0003-4584-5528.

Cite this article as: ÇIlıı B, Altın B, Aksoy S. Caffeine Enhances the Balance System and Postural Balance in Short Time in Healthy Individuals. Turk Arch Otorhinolaryngol 2021; 59(4): 253-60.

Conclusion: Consumption of an appropriate dose of caffeine may enhance body position, postural stability, and voluntary motor control ability. The combined use of the sensory organization test, the head shake sensory organization test, the adaptation test, and the limits of stability test in evaluating the balance mechanism in individuals with normal vestibular findings provides detailed information about postural sway.
\end{abstract}

Keywords: Postural balance, posturography, vestibular function tests, caffeine, psychoactive agents

Corresponding Author:

Bünyamin Çıldır; bunyamin.cildir@gmail.com

Received Date: 20.04 .2021

Accepted Date: 19.09.2021

Content of this journal is licensed under a Creative

Commons Attribution 4.0 International License.

Available online at www.turkarchotolaryngol.net 


\section{Introduction}

Caffeine, a xanthine alkaloid found in coffee, soft drinks, green tea, energy drinks, chocolate, and medications, is the most widely consumed psychoactive substance and central nervous system stimulant worldwide (1). While consumption of an appropriate dose of caffeine (200-300 mg) is beneficial for reducing physical fatigue, it causes increased wakefulness, and is good for improving motor performance, attention, and visual acuity (2). In a study by Liguori and Robinson (4), consumption of 200-400 mg of caffeine was reported to produce similar effects as alcohol consumption. Alcohol consumption, however, reduces balance stability, but such reduction is not generally associated with the effects of caffeine (3). Caffeine is readily absorbed by the body (approximately within 45 minutes), and reaches plasma concentration within 15-45 minutes in humans (4). Moreover, the half-life of caffeine ranges from 2.5 to 5 hours (5). Caffeine reaches various organ systems (e.g., the central nervous system, the peripheral system) after absorption and may reduce the nerve conduction velocity by blocking adenosine receptors (particularly A1 and A2) in these systems (2). The adenosine receptors in the organs are found in the hippocampus, the cerebral cortex, the somatosensory cortex, the cerebellum, and the hypothalamic nuclei (6). In the central nervous system, many sensorimotor systems (proprioceptive, visual, vestibular), such as the cerebellum and the brain stem, are necessary for balance (7). Among its various other tasks, the central nervous system is responsible for ensuring postural control in activities during the complex interaction between the neurological and the musculoskeletal systems $(8,9)$. At the same time, caffeine affects the cognitive function as it has three important mechanisms of action that have a psychostimulant effect on the central nervous system (10). Caffeine indirectly affects the release of neurotransmitters as it can act as a competitive antagonist against the depressant effects of adenosine (11). When this system is affected, mood, memory, alertness, cognitive function, and balance system are also affected.

While some studies have shown that the postural sway of healthy individuals had increased after caffeine intake despite the improvement of their physical endurance, others asserted that caffeine intake did not have any effect on postural sway (7, 12-14). Although there are studies conducted on the effects of caffeine (alcohol, energy drinks, etc.) on postural sway, most of the research questions (e.g., "Does caffeine increase/ decrease postural sway?" "Does caffeine have a placebo effect?" "How much does daily caffeine consumption affect the balance system?") have not yet been answered. While there are various methods of assessing a person's balance performance, posturography tests are the most common and the most effective tests to that end. Computerized dynamic posturography (CDP) is the gold standard, differentiating among sensory, motor, and central adaptive impairments of postural control (15).

Sensory Organization Test (SOT), Head Shake Sensory Organization Test (HS-SOT), Limits of Stability (LOS) Test, and Adaptation Test (ADT) are the subtests of CDP for assessing different parts of the vestibular system (16). SOT is one of the most widely used protocols for CDP, evaluating a person's ability to use the visual, vestibular, and somatosensory information required to sustain balance, and whether a person can maintain their body's center of gravity when the flow of visual and proprioceptive information is interrupted (17). Also, HS-SOT is more sensitive than SOT, especially in dizzy patients, because of the elimination of the somatosensory and visual systems and the addition of head shake movements to the standard SOT with the eyes closed (18).

This study aimed to investigate the effects of caffeine on the balance performance of healthy individuals. The extent to which caffeine affects postural sway and balance control and the effects of daily caffeine consumption on the balance function were determined in this study.

\section{Methods}

The study was approved by the Ethics Committee of Hacettepe University (approval no: GO 17/208) and conducted in compliance with the guidelines of the Declaration of Helsinki. All participants provided their informed consent for the study. All hearing and balance measurements were conducted at the Audiology Unit of Hacettepe University Hospital.

\section{Participants}

This study included 30 healthy subjects aged $18-35$ years. They were provided with detailed information about the study, after which their informed consent to participate in the study was obtained. Individuals who regularly consumed little $(\leq 100 \mathrm{mg} /$ day) or no caffeine were included in the study. Those who had any vestibular or neurological problems or a medical condition that would have impaired their balance and those who needed to avoid caffeine consumption due to health problems were not included. The participants were asked to fill out a medical history form and were given a list of caffeinated drinks that they were recommended not to consume at least 48 hours before each study session.

\section{Experimental Design}

The components of CDP (Neurocom International Inc., Clackamas, Oregon, USA) that were used to evaluate the study participants' balance performance were SOT, HSSOT, ADT, and LOS test. The participants were randomly assigned to one of the two groups: Group 1 was given two cups of specially prepared caffeinated coffee (300-350 mg) 
and Group 2 was given two cups of decaffeinated coffee prepared using the same method. Short breaks were given between tests. The tests were performed in two sessions per group. The coffee was given 45-60 minutes before the tests, and the participants were allowed to put additives in their coffee (milk and sugar or artificial sweeteners) if they did not want to drink black coffee. Also, the participants were not given any information about the type of their coffee and the amount of caffeine they consumed. In the first session, in Group 1, some individuals drank caffeinated, others did not; in Group 2, some of the individuals drank decaf, others did not drink at all. The second session was carried out after approximately 10 days, where the participants who had not drunk coffee in the first session drank coffee before the test and vice versa.

\section{Sensory Organization Test}

The SOT included six test positions, from easy to difficult (Figure 1). After the test procedure was explained to the participants, they were asked to wear a harness secured to the bars on the device and stand on a platform. Each of these six conditions was repeated three times to calculate the mean value of the measurements for the condition, and every trial lasted 20 seconds. The SOT equilibrium scores were the mean scores for the three trials. The mean equilibrium score for each condition and the composite equilibrium score for all the conditions were calculated within the 0-100 range according to the participant's postural sway at the limits of stability. A composite score of 0-59 indicated falls; 60-69, a risk of falling; and 70-100, normal results (19).

\section{Head Shake Sensory Organization Test}

HS-SOT is a modified test that was created by adding head shake movements to the standard SOT with the eyes closed. The use of somatosensory information is evaluated in condition 2 (C2), and vestibular inputs are tested in condition 5 (C5). The participants were asked to wear a gyro headband for detecting their movements and velocity, and to move their heads in the yaw, pitch, and roll planes in the $\mathrm{C} 2$ and $\mathrm{C} 5$ positions of SOT simultaneously with a rhythmic audio signal. The mean equilibrium score and equilibrium score ratio were used for the evaluation of the subjects in each plane (20).

\section{Adaptation Test}

The ADT measures the adaptation ability of the motor system by evaluating the automatic postural responses of a person to reaction forces. In this test, the subjects were asked to stand on the CDP platform and were exposed to five equivalent surface irregularities in two different conditions (i.e., toes up and toes down). The sway energy score for each of the conditions and the mean score for each of the toes-up and toes-down perturbations were calculated from the test results (19).

\section{Limits of Stability Test}

The LOS Test was performed on all subjects in eight directions (front, left-front, right-front, right, left, back, right-back, and left back). It is used to provide information about the subjects' abilities to move their center of gravity on a support surface using ankle strategies and weight transfer while staying in an upright position. The mean value of the eight completed trials for each target (front or back) was calculated to determine the five parameters of LOS: reaction time (RT), movement velocity (MV), endpoint excursion (EE), maximum excursion (ME), and directional control (DC) (16).

\section{Statistical Analysis}

IBM SPSS 23.0 was used for data analysis. Parametric tests were used because both Group 1 and Group 2 showed normal distributions. The paired-samples t-test was used to

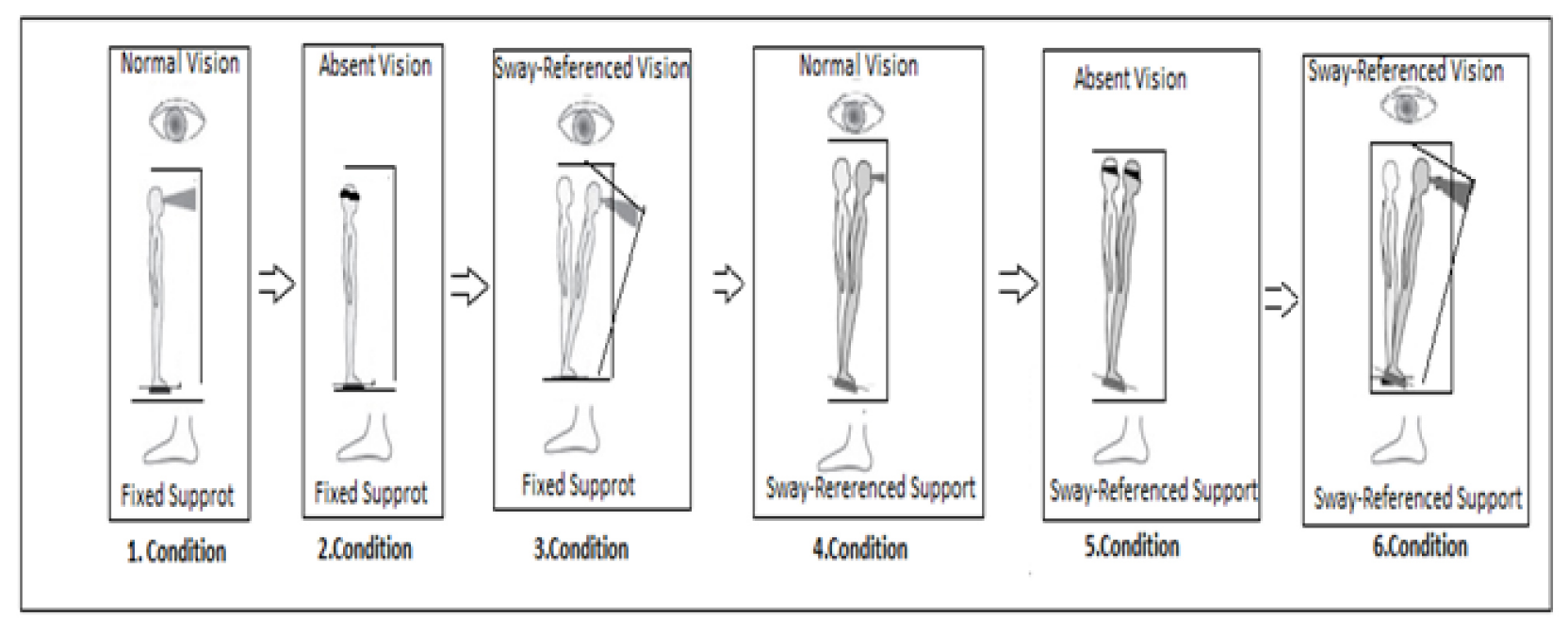

Figure 1. Six conditions of the Sensory Organization Test 
compare intragroup differences (before and after drinking coffee), and the independent-samples t-test was used to compare intergroup differences. Statistical significance was set at $<0.05$.

\section{Results}

The mean age of the 15 participants (seven males, eight females) in Group 1 who consumed caffeinated coffee was 23.66 years [range: $20-35$ years; standard deviation (SD): \pm 1.11 ], and the mean age of the 15 participants (eight males, seven females) in Group 2 who consumed decaffeinated coffee was 25.91 years (range: $20-35$ years; SD: \pm 1.59 ). All the balance scores before and after coffee intake for Group 1 and Group 2 were measured separately. The SOT, HS-SOT, $\mathrm{ADT}$, and LOS test mean scores of the participants, and the comparison data, are shown in Table 1.

\section{Differences between the SOT composite and equilibrium scores of the participants}

Regarding the participants' composite and balance scores from SOT (C2 and $\mathrm{C} 5)$, the $\mathrm{C} 5$ and composite balance scores of the participants in Group 1 showed a statistically significant increase after caffeine intake. In Group II, the $\mathrm{C} 5$ and composite balance scores of the participants who consumed decaffeinated coffee showed a statistically significant decrease $(p=0.001)$. There was no statistically significant difference between the $\mathrm{C} 2$ equilibrium scores of Group 1 ( $\mathrm{p}=0.199)$ and Group $2(\mathrm{p}=0.277)$ before and after coffee intake (Figure 2). The composite and mean $\mathrm{C} 5$ scores of the participants in both Group 1 and Group 2 are shown in Table 1.
Relationship between coffee intake (caffeinated and decaffeinated) and the HS-SOT and SOT scores

There was a statistically significant difference between the mean equilibrium scores of Group 1 in HS-SOT C5 in the vertical plane $(\mathrm{p}=0.001)$, horizontal plane $(\mathrm{p}=0.001)$, and roll plane $(\mathrm{p}=0.009)$ after coffee intake. In Group 2, after coffee intake, there was a statistically significant difference between the mean balance scores in HS-SOT C 5 in the vertical plane $(\mathrm{p}=0.009)$, horizontal plane $(\mathrm{p}=0.001)$, and roll plane $(\mathrm{p}=0.008)$, and between the mean balance scores in HS-SOT C2 in the vertical plane $(\mathrm{p}=0.046)$ and roll plane $(p=0.025)$. There was a statistically significant difference between the equilibrium scores of both groups in the vertical plane $(\mathrm{p}=0.001)$, horizontal plane $(\mathrm{p}=0.001)$, and roll plane $(\mathrm{p}=0.001)$ of HS-SOT C5 after coffee intake.

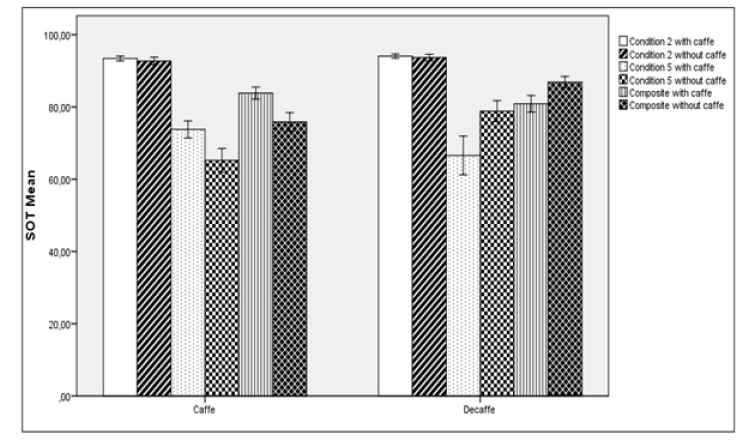

Figure 2. Results of the Sensory Organization Test, between groups comparison

SOT: Sensory Organization Test

Table 1. Comparison of Sensory Organization Test, Head Shake Test and Adaptation Test Scores between the two groups

\begin{tabular}{|c|c|c|c|c|c|c|}
\hline & $\begin{array}{l}\text { Group1 (with } \\
\text { caffeinated coffee) } \\
(\mathrm{n}=15)\end{array}$ & $\begin{array}{l}\text { Group } 1 \text { (without } \\
\text { coffee) } \\
(n=15)\end{array}$ & $\mathrm{p}$-value & $\begin{array}{l}\text { Group } 2 \text { (with } \\
\text { decaffeinated } \\
\text { coffee) } \\
(n=15)\end{array}$ & $\begin{array}{l}\text { Group } 2 \text { (without } \\
\text { coffee) } \\
(n=15)\end{array}$ & p-value \\
\hline SOT 2 & $93.31 \pm 1.60$ & $92.11 \pm 2.58$ & 0.411 & $94.08 \pm 1.08$ & $93.66 \pm 1.68$ & 0.479 \\
\hline SOT 5 & $71.88 \pm 6.22$ & $63.2 \pm 5.06$ & $0.001^{*}$ & $66.57 \pm 9.65$ & $78.84 \pm 5.25$ & $0.001^{*}$ \\
\hline HS-SOT 2(v) & $94.08 \pm 1.08$ & $93.66 \pm 1.68$ & 0.657 & $86.26 \pm 3.92$ & $87.95 \pm 3.61$ & $0.046^{*}$ \\
\hline HS-SOT 5(v) & $78.84 \pm 5.67$ & $66.57 \pm 9.65$ & $0.005^{*}$ & $47.28 \pm 12.65$ & $57.21 \pm 11.95$ & $0.009^{*}$ \\
\hline HS-SOT 2(h) & $90.85 \pm 2.28$ & $91.84 \pm 2.57$ & 0.127 & $92.84 \pm 1.38$ & $91.82 \pm 2.22$ & 0.090 \\
\hline HS-SOT 5(h) & $57.83 \pm 12.24$ & $37.41 \pm 11.61$ & $0.001^{*}$ & $54.93 \pm 7.2$ & $66.18 \pm 4.85$ & $0.001^{*}$ \\
\hline HS-SOT 2(r) & $88.64 \pm 2.66$ & $88.53 \pm 2.69$ & 0.065 & $91.35 \pm 2.2$ & $90.06 \pm 2.35$ & $0.025^{*}$ \\
\hline ADT Down & $43.73 \pm 10.19$ & $44.19 \pm 10.86$ & 0.637 & $43.26 \pm 8.27$ & $40.26 \pm 5.04$ & 0.073 \\
\hline
\end{tabular}




\section{Relationship between coffee intake and the ADT score}

There were no statistically significant differences between Group 1's ADT up scores ( $\mathrm{p}=0.886)$ and ADT down scores $(\mathrm{p}=0.637)$ or between Group 2's ADT up scores $(\mathrm{p}=0.551)$ and ADT down scores $(p=0.073)$ before and after coffee intake. While there was a statistically significant difference between the groups in the mean ADT up scores after coffee intake ( $p=0.042$ ), there was no significant difference between the groups in the mean ADT down scores ( $\mathrm{p}=0.196)$.

\section{Relationship between coffee intake and the LOS test score}

Regarding the mean values of the eight conditions in the LOS test parameters, a statistically significant decrease was observed in the endpoint excursion value after caffeine intake in Group 1 ( $\mathrm{p}=0.001)$.

Considering the LOS test parameters in Group 1 and Group 2 after coffee intake, a statistically significant difference was observed only in the endpoint travel parameter in the back direction $(\mathrm{p}=0.001)$ and the maximum travel parameter in the left direction $(p=0.007)$. No statistically significant differences were found among the reaction time $(\mathrm{p}=0.225)$, movement velocity parameters $(\mathrm{p}=0.127)$, and endpoint excursion parameters in the right direction $(p=0.074)$, left direction $(p=0.0245)$, and left-front direction $(p=0.610)$ in Group 1 after coffee intake (Table 2). Intragroup and intergroup comparisons of the different LOS test parameters are shown in Figure 3.

\section{Discussion}

In addition to their effects on the cognitive and cellular functions of the brain, caffeinated beverages are known to affect the audio-vestibular system. This study proved that healthy individuals who consume caffeinated coffee have significantly better postural control than those who do not consume caffeinated drinks or consume only decaffeinated coffee, particularly in conditions that do not involve difficult vestibular cues. Caffeine consumption increases wakefulness, improves the mood, and causes a release of catecholamines in the central nervous system, but it also impacts the heart, skeletal muscle, and adipocyte tissues (21).

Moreover, the aforementioned findings were thought to imply that a more effective use of the vestibular system could be achieved by caffeinated drink consumption despite the lack of visual support, and that caffeine intake could lead to more effective postural control. It has been stated that consumption of a moderate dose of caffeine can be effectively used as an ergogenic aid before exercise to increase the performance variables in various sports (22).

In addition, the SOT performance was found to be higher because the level of alertness was increased after caffeinated drink consumption, thereby leading to increased balance performance that would enable postural control as per voluntary motor control (23). McNerney et al. (24) stated that as caffeine enhances balance performance, it increases the level of alertness and the ability to focus on a task. In a previous study, however, the difference in the equilibrium scores between the caffeinated and non-caffeinated drink consumption sessions for individuals who consume large amounts of caffeine daily was higher than that for regular caffeine consumers.

Because the central and peripheral vestibular anomalies involving body balance may affect our ability to use vestibular

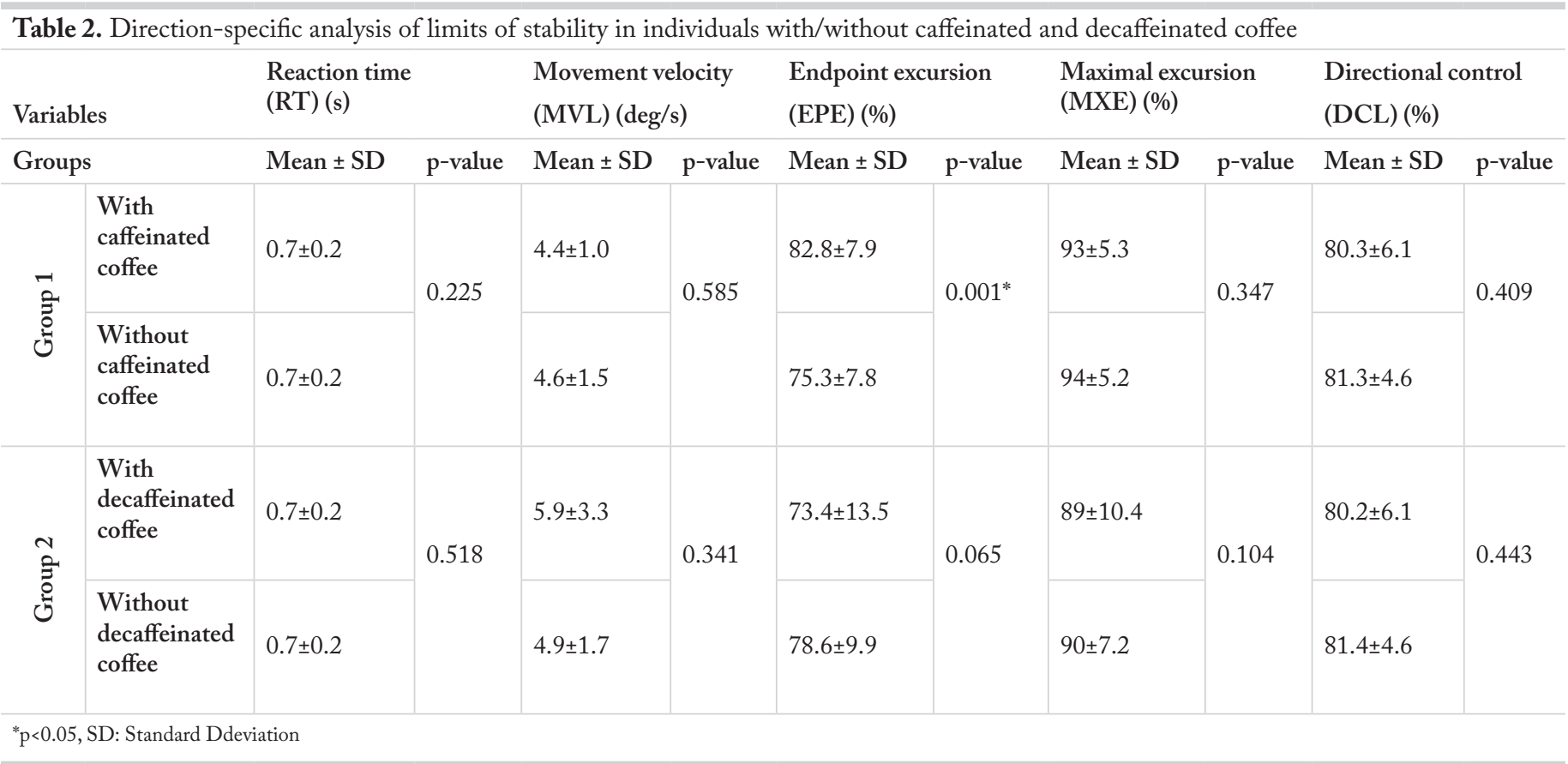


stimuli, increased sensory input to the central nervous system after caffeine intake is consistent with the results of our study which demonstrated the role of caffeine in postural balance (25). This finding is contrary to those of previous studies which reported that caffeine consumption did not affect postural balance, as the mean equilibrium scores (both when the eyes were open and were closed) minimally increased after caffeine intake (8).

However, it was surprising that the participants had lower mean equilibrium scores after consuming decaffeinated coffee than when they did not consume any drink. This could be a placebo effect because most of the participants had lower daily coffee consumption and caffeine is believed to negatively affect balance.

A study by Kim et al. (26), in which only static balance was measured, reported that the usual amount of caffeine intake influenced the postural stability of stroke patients using the somatosensory function by reducing the visual deprivation effect. In this study, the static and dynamic balance performances were also evaluated with the SOT, HS-SOT, ADT, and LOS test. The LOS test is a reliable tool for

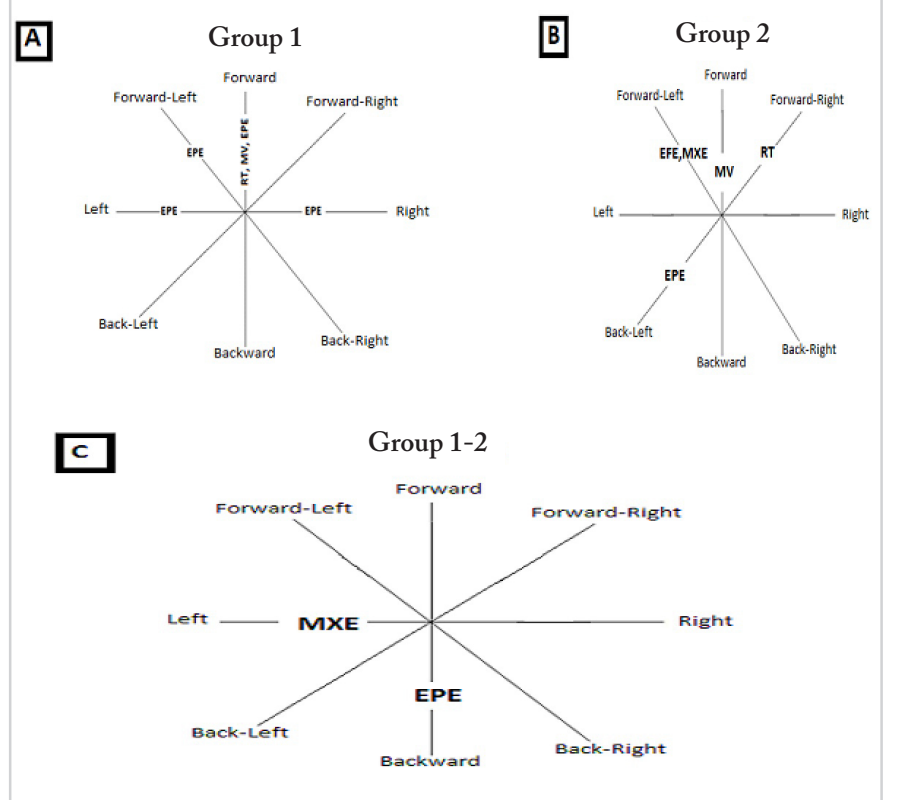

Figure 3. Limits of Stability Test Results: (a) differences parameters and locations in Limits of Stability Test, between before and after consuming coffee for Group 1; (b) parameters and locations in Limits of Stability Test, between before and after consuming coffee for Group 2; (c) differences parameters and locations in Limits of Stability Test between groups. The Wilcoxon Test was used for ingroup comparison and the Mann-Whitney U Test was used for comparison between groups.

EPE: Endpoint excursion, MXE: Maximum excursion, MV: Velocity of movement, RT: Reaction time

Forward, backward, right, left, forward-right, forward-left, back-right and back-left shows shift in eight different directions assessing a person's voluntary motor control skills in terms of the body's center of gravity and body position (27). Juras et al. (28) conducted a study with healthy individuals and found that the most reliable LOS test parameter for assessing the body's ability to control its center of gravity is excursion. In the presented study, there was a statistically significant difference in the endpoint and maximum excursion (\%) values, as observed in the comparison of the LOS test results before and after caffeinated and decaffeinated drink consumption. Moreover, there was a statistically significant increase in the endpoint and maximum excursion (\%) values from baseline in the participants who consumed caffeinated drink than in those who consumed decaffeinated drink. As the differences in endpoint and maximum excursion values can be observed during the most complex movements of the body, it is thought that caffeine has a positive impact on the balance system during dynamic movements against the center of gravity (16).

We found that the HS-SOT results were significantly better in all the planes in the participants who consumed caffeinated drink than in those who consumed decaffeinated drink, which also demonstrated the positive effect of caffeine intake on the dynamic balance system despite the addition of head shake movements. Interestingly, this study, to date, is the only one that investigated the impact of caffeine intake on HS-SOT performance.

The head shake test leads to an increased neural activity especially in those with unilateral peripheral vestibular weakness, even though they have normal results in the standard tests, and thereby facilitates the diagnosis in such individuals (18). As the active head movement in SOT C2 and C5 may lead to disruption in postural control and the body's compensation mechanism, even small differences in postural balance are easily detectable (29). One of the most important advantages of the head shake test is the simultaneous stimulation of the peripheral vestibular system with postural control (30).

The ADT, the least reliable among posturography tests assessing motor skills, can be influenced by other factors not associated with balance, such as anxiety, fear, and biomechanical factors (15). Although this test was a part of the SOT, its results were not compared with those of the other tests because its parameters are not comparable with those of other test batteries (19). Also, as there was no significant difference between the mean ADT scores of the groups in this study, we conclude that ADT is not an effective tool for evaluating the effects of caffeine intake on balance. Taken together, these findings suggest that the SOT, the HS-SOT, and the LOS test are beneficial tools for determining the effects of caffeine intake on functional balance. It was also thought that consuming two cups of coffee daily would have positive impacts on balance and attention in individuals with normal vestibular findings. 
This study has several limitations, including the small number of participants and the fact that different doses of caffeine were not used. Also, as we did not check the body mass index (BMI) of the participants, we do not know whether the intake of the same dose of caffeine always has the same effect on the subject.

\section{Conclusion}

The consumption of an appropriate amount of caffeine can enhance postural control under different conditions because caffeine increases attention and provides stimulation. This study is important in that it showed the positive effects of caffeine consumption on static and dynamic balance performance. It was found that the effect of caffeine consumption, particularly on balance performance, could be evaluated with the SOT and the HS-SOT. Future studies investigating the effects of the consumption of different amounts of caffeine and the BMI of the participants on the balance system will add valuable contributions to the literature.

Ethics Committee Approval: Ethics committee approval was received for this study from the Ethics Committee of Hacettepe University Non-Interventional Clinical Trials (GO 17/208).

Informed Consent: Written informed consent was obtained from trainers and trainees who participated in this study.

Peer-review: Externally peer-reviewed.

\section{Authorship Contributions}

Concept: B.Ç., B.A., Design: B.Ç., B.A., Supervision: B.Ç., B.A., S.A., Data Collection and/or Processing: B.Ç., B.A., Analysis and/or Interpretation: B.Ç., B.A., S.A., Literature Search: B.Ç., B.A., Writing: B.Ç., B.A., Critical Review: B.Ç., B.A., S.A.

Conflict of Interest: The authors have no conflicts of interest to declare.

Financial Disclosure: The authors declared that this study has received no financial support.

\section{Main Points}

- Caffeine has a stimulating effect on posture and automatic motor control.

- A certain amount of caffeine consumption has a positive impact on the balance system during dynamic movements against the center of gravity.

- Head shake-sensory organization test and limit of stability test are useful tests used for the detailed evaluation of the balance mechanism.

\section{References}

1. Seifert SM, Schaechter JL, Hershorin ER, Lipshultz SE. Health effects of energy drinks on children, adolescents, and young adults. Pediatrics 2011; 127: 511-28. [Crossref]

2. Smith A. Effects of caffeine on human behavior. Food Chem Toxicol 2002; 40: 1243-55. [Crossref]

3. Dixit A, Vaney N, Tandon OP. Effect of caffeine on central auditory pathways: an evoked potential study. Hearing Res 2006; 220: 61-6. [Crossref]

4. Liguori A, Robinson JH. Caffeine antagonism of alcohol-induced driving impairment. Drug Alcohol Dep 2001; 63: 123-9. [Crossref]

5. Fredholm BB, Bättig K, Holmén J, Nehlig A, Zvartau EE. Actions of caffeine in the brain with special reference to factors that contribute to its widespread use. Pharmacological Rev 1999; 51: 83-133. [Crossref]

6. Culm-Merdek KE, von Moltke LL, Harmatz JS, Greenblatt DJ. Fluvoxamine impairs single-dose caffeine clearance without altering caffeine pharmacodynamics. Br J Clin Pharmacol 2005; 60: 486-93. [Crossref]

7. Dias RB, Rombo DM, Ribeiro JA, Henley JM, Sebastião AM. Adenosine: setting the stage for plasticity. Trends Neurosci 2013; 36: 248-57. [Crossref]

8. Enriquez A, Sklaar J, Viirre E, Chase B. Effects of caffeine on postural stability. Int Tinnitus J 2009; 15: 161-3. [Crossref]

9. Rosario MG, Collazo H, Mateo M, Gonzalez-Sola M, Bayron F. Increased static postural sway after energy drink consumption: a randomized trial. F1000Research 2017; 6: 2036. [Crossref]

10. Shumway-Cook A, Woollacott MH, editors. Motor control: translating research into clinical practice. 3rd ed. Philadelphia: Lippincott Williams \& Wilkins; 2007. [Crossref]

11. Fiani B, Zhu L, Musch BL, Briceno S, Andel R, Sadeq N, et al. The neurophysiology of caffeine as a central nervous system stimulant and the resultant effects on cognitive function. Cureus. 2021; 13; e15032. [Crossref]

12. Sebastião AM, Ribeiro JA. Adenosine receptors and the central nervous system. Handb Exp Pharmacol 2009: 471-534. [Crossref]

13. Norager CB, Jensen MB, Madsen MR, Laurberg S. Caffeine improves endurance in 75-yr-old citizens: a randomized, doubleblind, placebo-controlled, crossover study. J Appl Physiol 2005; 99: 2302-6. [Crossref]

14. Lee J. Effects of caffeine of coffee on fine motor, gross motor and balance in healthy adult male. Daejeon: Chungnam National University, Dissertation, 2007. [Crossref]

15. Trueblood PR, Rivera M,Lopez C,Bentley C,Wubenhorst, N.Agebased normative data for a computerized dynamic posturography system that uses a virtual visual surround environment. Acta Otolaryngol 2018; 138: 597-602. [Crossref] 
16. Faraldo-García A, Santos-Pérez S, Rossi-Izquierdo M, LirolaDelgado A, Vaamonde-Sánchez-Andrade I, Del-Río-Valeiras M, et al. Posturographic limits of stability can predict the increased risk of falls in elderly patients with instability? Acta Otolaryngol 2016; 136: 1125-9. [Crossref]

17. Black FO. What can posturography tell us about vestibular function? Ann N Y Acad Sci 2001; 942: 446-64. [Crossref]

18. Altın B, Aksoy S. Clinical significance of head shake movement in three planes in individuals with dizziness. Am J Otolaryngol 2020; 41: 102308. [Crossref]

19. Oda DTM, Ganança CF. Computerized dynamic posturography in the assessment of body balance in individuals with vestibular dysfunction. Audiol Commun Res 2015; 20: 89-95. [Crossref]

20. Honaker JA, Janky KL, Patterson JN, Shepard NT. Modified head shake sensory organization test: sensitivity and specificity. Gait Posture 2016; 49: 67-72. [Crossref]

21. Barcelos RP, Lima FD, Carvalho NR, Bresciani G, Royes LF. Caffeine effects on systemic metabolism, oxidative-inflammatory pathways, and exercise performance. Nutr Res 2020; 80: 1-17. [Crossref]

22. Salinero JJ, Lara B, Del Coso J. Effects of acute ingestion of caffeine on team sports performance: a systematic review and meta-analysis. Res Sports Med 2019; 27: 238-56. [Crossref]

23. Lacour M, Bernard-Demanze L, Dumitrescu M. Posture control, aging, and attention resources: models and posture-analysis methods. Neurophysiol Clin 2008; 38: 411-21. [Crossref]
24. McNerney KM, Coad ML, Burkard RF. The influence of caffeine on the sensory organization test. J Am Acad Audiol 2014; 25: 5218. [Crossref]

25. Asai M, Watanabe Y, Ohashi N, Mizukoshi K. Evaluation of vestibular function by dynamic posturography and other equilibrium examinations. Acta Otolaryngol Suppl 1993; 113: 120-4. [Crossref]

26. Kim, W. S., Choi, C. K., Yoon, S. H., \& Kwon, J. Y. Usual dose of caffeine has a positive effect on somatosensory related postural stability in hemiparetic stroke patients. Ann Rehabil Med 2014; 38: 775-83. [Crossref]

27. Ayhan C, Bilgin S, Aksoy S, Yakut Y. Functional contributors to poor movement and balance control in patients with low back pain: a descriptive analysis. J Back Musculoskelet Rehabil 2016; 29: 47786. [Crossref]

28. Juras G, Słomka K, Fredyk A, Sobota G, Bacik B. Evaluation of the limits of stability (LOS) balance test. J Hum Kinet 2008; 19: 39-52. [Crossref]

29. Paloski WH, Wood SJ, Feiveson AH, Black FO, Hwang EY, Reschke MF. Destabilization of human balance control by static and dynamic head tilts. Gait Posture 2006; 23: 315-23. [Crossref]

30. Peters JF. Computerized dynamic posturography (CDP) and the assessment of balance with active head movements. J Korean Balance Soc 2007; 6: 243-7. [Crossref] 